\title{
Papers
}

\section{Hospital bed utilisation in the NHS, Kaiser Permanente, and the US Medicare programme: analysis of routine data}

Chris Ham, Nick York, Steve Sutch, Rob Shaw

\begin{abstract}
Objective To compare the utilisation of hospital beds in the NHS in England, Kaiser Permanente in California, and the Medicare programme in the United States and California. Design Analysis of routinely available data from 2000 and 2001 on inpatient admissions, lengths of stay, and bed days in populations aged over 65 for 11 leading causes of use of acute beds.

Setting Comparison of NHS data with data from Kaiser Permanente in California and the Medicare programme in California and the United States; interviews with Kaiser Permanente staff and visits to Kaiser facilities.

Results Bed day use in the NHS for the 11 leading causes is three and a half times that of Kaiser's standardised rate, almost twice that of the Medicare California's standardised rate, and more than 50\% higher than the standardised rate in Medicare in the United States. Kaiser achieves these results through a combination of low admission rates and relatively short stays. The lower use of bed days in Medicare in California compared with Medicare in the United States suggests there is a "California effect" as well as a "Kaiser effect" in hospital utilisation.

Conclusion The NHS can learn from Kaiser's integrated approach, the focus on chronic diseases and their effective management, the emphasis placed on self care, the role of intermediate care, and the leadership provided by doctors in developing and supporting this model of care.
\end{abstract}

\section{Introduction}

Feachem and colleagues have compared the costs and performance of the NHS and the health maintenance organisation Kaiser Permanente in California. ${ }^{1}$ After adjusting for age differences in the populations served, they reported that the NHS used three times the number of acute bed days as Kaiser.

Feachem and colleagues focused on aggregate differences in use of bed days. To explore the issues raised in their analysis further, we took a number of the leading causes of bed day use in the NHS and compared resource utilisation for each cause. In so doing, we sought to understand how Kaiser is able to limit the use of beds for conditions such as stroke and hip fracture, which are a major source of demand on NHS hospitals.

We concentrated on people aged 65 and over because older people make the greatest use of acute beds. Also, focus on this age group enables the comparison between the NHS and Kaiser to be located in the context of the utilisation of services by the Medicare population for the United States as a whole and in California.
Throughout the paper we use the term Kaiser as shorthand for the Kaiser Permanente Medical Care Programme. The programme is made up of the Kaiser Foundation Health Plan, Kaiser Foundation Hospitals, and the Permanente Medical Groups. There are more than 10000 Permanente physicians in the medical groups and they serve more than 8 million Kaiser Permanente members. Kaiser is one of the oldest established health plans in the United States; it uses a range of managed care techniques (see below) to ensure that resources are used efficiently.

\section{Methods}

We used routinely available data to identify 15 leading causes of acute hospital admission and bed day use in the NHS in England. These data were drawn from the hospital episodes statistics for 2000-1. We excluded two healthcare resource groups, (invalid primary diagnosis and ill defined signs and symptoms), which are important causes of acute bed day use but seemed unlikely to yield meaningful comparisons, and two others (cataract surgery and cardiac catheterisation) where patients are usually treated as day cases. Table 1 shows that the remaining 11 healthcare resource groups account for $11.5 \%$ of total bed day use in the NHS. To assess the extent to which bed day use for these 11 healthcare resource groups is representative of all causes of admission, we compared our results with the aggregate analysis undertaken by Feachem and colleagues.

Data from Kaiser were drawn from Kaiser's cost management information system and are for the year 2000. Medicare data were drawn from the $5 \%$ analytic file (inpatient) for beneficiaries in fee for service Medicare and are also for the year 2000. The population for Kaiser was the membership aged 65 and over. For Medicare, the population included all beneficiaries aged 65 and over, living in the United States, with parts A and B coverage, in fee for service plans.

To match as closely as possible the definitions used by Kaiser and Medicare we mapped healthcare resource groups against diagnosis related groups, using the NHS Wales activity database, which contains both healthcare resource groups and diagnosis related groups. The mapping was specified to diagnosis level (ICD-10; international classification of diseases, 10th edition) for medical groupings, and procedure level (OPCS/ICD-9CM) for surgical groupings to ensure the groupings were clinically similar. Where necessary, less specific groupings were split (table 2).

NHS data excluded lengths of stay of more than 365 days and used provider spells rather than finished consultant episodes. Provider spells were chosen to enable a closer match to the definition used for inpatients in the Kaiser and Medicare data. 
Table 1 Selected healthcare resource groups-11 leading causes of use of NHS bed days (England, 2000-1) ${ }^{2}$

\begin{tabular}{|c|c|c|}
\hline Group & No $(\%)$ total bed days & Bed days per 1000 population \\
\hline A22 Non-transient stroke or cerebrovascular accident $>69$ or with complications & $1040324(2.0)$ & 20.8 \\
\hline H33 Neck of femur fracture $>69$ or with complications & $740000(1.5)$ & 14.8 \\
\hline D20 Chronic obstructive pulmonary disease or bronchitis & $713416(1.4)$ & 14.3 \\
\hline D21 Asthma $>49$ or with complications & $599583(1.2)$ & 12.0 \\
\hline E18 Heart failure or shock $>69$ or with complications & $590356(1.2)$ & 11.8 \\
\hline L09 Kidney or urinary tract infections $>69$ or with complications & $470240(0.9)$ & 9.4 \\
\hline H02 Primary hip replacement & $422099(0.8)$ & 8.4 \\
\hline E12 Acute myocardial infarction without complications & $416254(0.8)$ & 8.3 \\
\hline H04 Primary knee replacement & $354866(0.7)$ & 7.1 \\
\hline E33 Angina $>69$ or with complications & $345977(0.7)$ & 6.9 \\
\hline E04 Coronary bypass & $196141(0.4)$ & 3.9 \\
\hline Total & $5889256(11.5)$ & \\
\hline
\end{tabular}

Healthcare resource groups were ranked according to bed days used in the NHS and the leading causes of admissions and day case rates were also examined. "Invalid primary diagnosis" and "ill defined signs and symptoms" were excluded from the list of leading causes of bed day use, resulting in the selection of the 11 causes in this table for analysis.

We took differences in the age structure of the populations studied into account by standardising Kaiser and Medicare data to populations in England. Age specific rates for each five year age band from these data were multiplied by population weights from England; for hospital admissions we used the resident population for the standardisation. For length of stay we used the population admitted to hospital. Bed days were standardised by multiplying the adjusted figures for admission rates and lengths of stay.

To understand the reasons for differences in bed day use between the NHS and Kaiser, one of us $(\mathrm{CH})$ visited California and interviewed senior clinical and managerial staff in Kaiser's headquarters and in one of its medical facilities. This was followed by a further visit in which 35 clinicians and managers from the NHS saw at first hand Kaiser's facilities and services. The results of this qualitative component of the study were tested and discussed with Kaiser staff, who confirmed that they represented an accurate account of Kaiser's approach (B Crane, personal communication, April 2003).

\section{Results}

For the 11 causes selected for analysis, we have data on admission rates, length of stay, and bed day use for inpatients. The main comparisons are between the NHS and Kaiser. Data for Medicare are included to provide context for these comparisons.

\section{Admission rates}

Table 3 shows the comparison of admission rates. For most causes, though not all, crude admission rates are higher in the NHS than in Kaiser. The biggest differences in admission rates are for angina and for bronchitis or asthma; NHS rates are four to five times higher than in Kaiser. Admission rates in Kaiser are higher for heart failure or shock, acute myocardial infarction, knee replacements, and kidney or urinary tract infection. Standardised admission rates display a similar pattern.

Medicare admission rates (crude and standardised) in both California and the United States are generally higher than in the NHS. The exceptions are angina and bronchitis or asthma.

\section{Lengths of stay}

Table 4 shows the comparison of lengths of stay. For all causes, crude lengths of stay are higher in the NHS than in Kaiser. The biggest differences in lengths of stay are for stroke and hip fracture; NHS stays are five to six times higher than in Kaiser.

Standardised lengths of stay are higher in the NHS than in Kaiser (table 4). For all causes, standardised lengths of stay for Kaiser are similar to the crude averages. This reflects the flat distribution of length of stay by age in the Kaiser data, unlike in the NHS, where there is a clear and positive relation between age and length of stay.

Medicare lengths of stay (crude and standardised) in both California and the United States are all shorter than in the NHS and usually longer than in Kaiser.

\section{Bed days}

For all causes, crude bed day use is higher in the NHS than in Kaiser (table 5). The biggest differences are for angina and for bronchitis or asthma; NHS bed day utilisation is 14 to 15 times higher than in Kaiser. Standardised bed days display a similar pattern.

\section{Table 2 NHS-Kaiser mapping}

Group

Specific cerebrovascular disorder except transient ischaemic attack

Neck of femur fracture-with hip/femur procedure

Chronic obstructive pulmonary disease excluding bronchitis

Heart failure and shock

Bronchitis and asthma (Kaiser data excludes patients aged <17)

Kidney or urinary tract infections

Acute myocardial infarction

Angina pectoris

Primary hip replacement

Primary knee replacement

Coronary bypass
Diagnosis related groups included (Kaiser/Medicare data)
Healthcare resource groups included (NHS data) A19, A22, A23, A99* H33t, H34t, H99†

\begin{tabular}{cc}
$210-212$ & $\mathrm{H} 33 \dagger, \mathrm{H} 34 \dagger, \mathrm{H} 99 \dagger$ \\
\hline 088 & $\mathrm{D} 20^{\star}$ \\
\hline $6,097,101,102$ & $\mathrm{E} 18, \mathrm{E} 19$ \\
\hline 320,321 & $\mathrm{D} 20^{\star}, \mathrm{D} 21, \mathrm{D} 22$ \\
\hline $121-123$ & $\mathrm{~L} 09, \mathrm{~L} 10, \mathrm{~L} 21^{\star}$ \\
\hline 140 & $\mathrm{E} 11, \mathrm{E} 12$ \\
\hline $209 \ddagger$ & $\mathrm{E} 33, \mathrm{E} 34$ \\
\hline $209 \ddagger$ & $\mathrm{H} 02$ \\
\hline 106,107 & $\mathrm{H} 04$ \\
\hline
\end{tabular}

* Subset of the healthcare resource group used based on ICD-10 diagnoses consistent with US diagnosis related group definition.

†Subset of the healthcare resource group used based on OPCS procedures consistent with US diagnosis related group definition.

$\ddagger$ Subset of the diagnosis related group used based on ICD-9CM procedures consistent with UK healthcare resource group definition. 
Table 3 Number of inpatient admissions (per 100000 population) in people aged over 65

\begin{tabular}{|c|c|c|c|c|c|c|c|}
\hline \multirow[b]{2}{*}{ Group } & \multirow[b]{2}{*}{ NHS } & \multicolumn{2}{|c|}{ Kaiser } & \multicolumn{2}{|c|}{ Medicare California } & \multicolumn{2}{|c|}{ Medicare United States } \\
\hline & & Unstandardised & Standardised & Unstandardised & Standardised & Unstandardised & Standardised \\
\hline Stroke & 823 & 712 & 788 & 1201 & 1155 & 1212 & 1183 \\
\hline Chronic obstructive pulmonary disease & 699 & 536 & 558 & 1081 & 1067 & 1262 & 1256 \\
\hline Bronchitis or asthma & 531 & 129 & 141 & 231 & 225 & 318 & 310 \\
\hline Coronary bypass & 144 & 103 & 97 & 289 & 296 & 313 & 321 \\
\hline Acute myocardial infarction & 550 & 836 & 893 & 702 & 675 & 942 & 923 \\
\hline Heart failure/shock & 556 & 1008 & 1118 & 1966 & 1893 & 2332 & 2272 \\
\hline Angina pectoris & 783 & 146 & 152 & 180 & 176 & 205 & 203 \\
\hline Hip replacement & 342 & 250 & 256 & 622 & 602 & 661 & 644 \\
\hline Knee replacement & 344 & 373 & 367 & 479 & 479 & 557 & 557 \\
\hline Hip fracture & 315 & 311 & 388 & 516 & 489 & 562 & 535 \\
\hline Kidney or urinary infection & 396 & 449 & 526 & 762 & 726 & 736 & 708 \\
\hline
\end{tabular}

Medicare bed day use (crude and standardised) in both California and the United States is usually lower than in the NHS. The exceptions are for coronary bypass and for heart failure or shock.

\section{Overall utilisation}

For the 11 causes selected for study, total bed day use in the NHS is three and a half times that of Kaiser's standardised rate, almost twice that of the Medicare California standardised rate, and over $50 \%$ higher than the standardised rate in Medicare in the United States. Kaiser achieves these results through a combination of low admission rates and short lengths of stay in hospital. By contrast, Medicare has high admission rates and relatively short stays. The NHS has long stays and admission rates that tend to come between those of Medicare and Kaiser.

\section{Discussion}

The overall differences between the NHS and Kaiser for these 11 healthcare resource groups are consistent with the findings of Feachem and colleagues on aggregate bed day use for all healthcare resource groups. This suggests that these 11 causes are not unrepresentative of others. However, in contrast to Feachem and colleagues, we found that differences in lengths of stay are more important in accounting for overall differences in bed day use than differences in admission rates. This is probably because our approach focused on the leading causes of NHS bed day use.

The Medicare data included in our analysis allow the performance of Kaiser to be viewed in a wider context. Our results indicate that aspects of Kaiser's approach to health care contribute to its performance over and above the impact of payment methods and managed care approaches prevalent in the United States.
Our data also suggest that there is a "California effect" in the way in which acute beds are used. The fact that Kaiser is a major insurer and provider in California may help in part to explain lower bed day use in Medicare in California than in Medicare in the United States.

\section{Limitations}

Three limitations should be noted. Firstly, differences in coding or recording may be affecting some of the comparisons. It seems that a relatively high proportion of heart patients admitted in the NHS are recorded as having angina, whereas in Kaiser and Medicare more patients would be recorded under heart failure or shock or under acute myocardial infarction. Similar issues arise in relation to bronchitis or asthma and chronic obstructive pulmonary disease.

Secondly, NHS data exclude the use of beds in the private sector. In England, private hospital beds are used mainly as a way of avoiding NHS queues by patients waiting for elective surgical procedures such as hip replacements and knee replacements. Omission of these data means that admission rates and bed day use for some health resource groups are understated in our analysis.

Thirdly, the NHS data presented here do not distinguish between the time that patients spend in an acute hospital and the time they spend in a community hospital or similar facility. This is a limitation of the reporting of activity data in the hospital episodes statistics system and means that the NHS figures overestimate the use of acute beds in comparison with Kaiser and Medicare.

The net effect of the exclusion of the use of private beds and inclusion of the use of community hospitals on bed day use in the NHS is not known.

Table 4 Length of hospital stay (days) for people aged over 65

\begin{tabular}{|c|c|c|c|c|c|c|c|}
\hline \multirow[b]{2}{*}{ Group } & \multirow[b]{2}{*}{ NHS } & \multicolumn{2}{|c|}{ Kaiser } & \multicolumn{2}{|c|}{ Medicare California } & \multicolumn{2}{|c|}{ Medicare United States } \\
\hline & & Unstandardised & Standardised & Unstandardised & Standardised & Unstandardised & Standardised \\
\hline Stroke & 27.08 & 4.29 & 4.26 & 5.84 & 5.84 & 6.54 & 6.53 \\
\hline Chronic obstructive pulmonary disease & 9.87 & 3.82 & 3.79 & 5.43 & 5.35 & 5.42 & 5.37 \\
\hline Bronchitis or asthma & 11.73 & 3.11 & 3.09 & 4.05 & 4.22 & 4.41 & 4.41 \\
\hline Coronary bypass & 13.27 & 9.82 & 9.60 & 8.86 & 8.63 & 10.37 & 9.98 \\
\hline Acute myocardial infarction & 9.39 & 4.37 & 4.35 & 5.22 & 5.14 & 5.60 & 5.46 \\
\hline Heart failure or shock & 12.42 & 3.72 & 3.70 & 5.29 & 5.28 & 5.39 & 5.37 \\
\hline Angina pectoris & 5.88 & 2.22 & 2.21 & 2.66 & 2.58 & 2.62 & 2.56 \\
\hline Hip replacement & 12.60 & 4.52 & 4.54 & 5.71 & 5.41 & 5.69 & 5.46 \\
\hline Knee replacement & 11.32 & 4.16 & 4.17 & 4.52 & 4.54 & 4.39 & 4.40 \\
\hline Hip fracture & 26.88 & 4.94 & 4.89 & 5.99 & 5.97 & 6.48 & 6.47 \\
\hline Kidney or urinary tract infection & 15.19 & 3.78 & 3.80 & 5.14 & 5.11 & 5.31 & 5.32 \\
\hline
\end{tabular}


Table 5 Number of bed days per 100000 population aged over 65

\begin{tabular}{|c|c|c|c|c|c|c|c|}
\hline \multirow[b]{2}{*}{ Group } & \multirow[b]{2}{*}{ NHS } & \multicolumn{2}{|c|}{ Kaiser } & \multicolumn{2}{|c|}{ Medicare California } & \multicolumn{2}{|c|}{ Medicare United States } \\
\hline & & Unstandardised & Standardised & Unstandardised & Standardised & Unstandardised & Standardised \\
\hline Stroke & 22289 & 3053 & 3358 & 7012 & 6750 & 7930 & 7726 \\
\hline Chronic obstructive pulmonary disease & 6906 & 2046 & 2118 & 5874 & 5709 & 6833 & 6747 \\
\hline Bronchitis or asthma & 6224 & 402 & 435 & 936 & 951 & 1403 & 1368 \\
\hline Coronary bypass & 1915 & 1006 & 935 & 2562 & 2552 & 3250 & 3205 \\
\hline Acute myocardial infarction & 5166 & 3651 & 3886 & 3667 & 3470 & 5278 & 5040 \\
\hline Heart failure or shock & 6905 & 3746 & 4137 & 10403 & 9999 & 12569 & 12191 \\
\hline Angina pectoris & 4600 & 325 & 334 & 478 & 454 & 538 & 520 \\
\hline Hip replacement & 4314 & 1132 & 1161 & 3552 & 3258 & 3762 & 3514 \\
\hline Knee replacement & 3893 & 1553 & 1533 & 2164 & 2172 & 2444 & 2448 \\
\hline Hip fracture & 8455 & 1536 & 1899 & 3094 & 2921 & 3639 & 3460 \\
\hline Kidney or urinary tract infection & 6010 & 1698 & 2000 & 3914 & 3712 & 3906 & 3767 \\
\hline
\end{tabular}

\section{Integration}

The qualitative study we undertook found that the most distinctive feature of the Kaiser model is the way in which it integrates care. The model contains three important aspects of integration.

Firstly, Kaiser integrates funding with the provision of care in that as an insurer it collects premiums from members and as a provider it delivers care to these members. Providers know that they have to work within the envelope of resources earned by the insurance plan. Physicians in the Permanente medical groups have an interest in minimising hospital stays because they share responsibility for the success of the programme.

Secondly, Kaiser integrates inpatient care and outpatient care. This enables patients to move easily between hospitals and the community, or into skilled nursing facilities should care in these facilities be needed. Medical specialists are uncoupled from the hospital and work alongside generalists in multispecialty medical groups. Specialists have no incentive to admit patients to hospital or keep them in longer than is appropriate.

Thirdly, Kaiser integrates prevention, diagnosis, treatment, and care. This is most apparent in relation to management of chronic disease-for example, for patients with heart failure or asthma. Care for patients with these conditions is delivered within the framework of evidence based clinical guidelines and is actively managed at all stages. Doctors who work for Kaiser also have fast access to diagnostic services in the outpatient setting, thereby avoiding patients staying in hospital.

\section{Managed care}

When patients are admitted to hospital, there is a strong emphasis on minimising stays and maintaining the flow of patients through the hospital through the use of managed care techniques. For example, care pathways have been developed for patients undergoing hip replacements and knee replacements, specifying what should happen on each day of hospital treatment. Kaiser employs specialist discharge staff to ensure that patients are not kept in hospital unnecessarily. This avoids the practice of patients lying in wait for discharge, so familiar in the NHS. ${ }^{3}$

Patients are enabled to return home by being supported to do as much as possible for themselves. Orthopaedic patients are therefore taught how to dress themselves, the exercises they need to do, and how to take drugs such as anticoagulants in the home. By offering advice and support in person and by telephone and by managing the expectations of patients and families, Kaiser staff enable hospitals to be used only when necessary.

Skilled nursing facilities play a part in accounting for the much shorter lengths of stay in Kaiser. Nurses and therapists are closely involved in providing care in a skilled nursing environment and offer an intensity of support that allows patients to be discharged home as soon as appropriate.

Two characteristics of the Kaiser model-one internal, one external-enable care to be delivered in this way. The internal characteristic is the leadership provided by Permanente physicians in developing and supporting this model of care. ${ }^{4}$ The external characteristic is the market environment in which Kaiser operates. The ever present threat of members leaving the health plan means that Kaiser must be responsive to its membership by offering accessible services to a high standard and at a reasonable cost. ${ }^{5}$

\section{Differences between Kaiser and the NHS}

Three differences between the NHS and Kaiser may affect the transferability of aspects of the Kaiser model. Firstly, as Feachem and colleagues reported, Kaiser has considerably more

\section{What is already known on the topic}

Kaiser Permanente in California uses far fewer acute bed days in relation to the population served than the NHS

The integrated model of care used in Kaiser Permanente explains its ability to keep patients out of hospital and to provide care in the community

Debate continues about the comparative costs of providing care in the NHS and Kaiser and differences in the characteristics of the populations served

\section{What this study adds}

The NHS uses three and a half times the number of acute bed days as Kaiser Permanente for 11 leading causes of bed day use in the NHS

The NHS uses twice the number of acute bed days as Medicare in California and 50\% more than Medicare in the United States for these causes

Differences in length of stay are more important than differences in admission rates in explaining variations in bed day use for the conditions selected in this study

Kaiser Permanente achieves lower utilisation of acute bed days through integration of care, active management of patients, the use of intermediate care, self care, and medical leadership 
specialists per 100000 population than the NHS-for example, twice the concentration of gynaecologists and three times the concentration of cardiologists. It is likely that the availability of extra specialists contributes to the differences we have observed. One hypothesis would be that there is a substitution effect between beds and staff, with the NHS having to make greater use of beds because it employs fewer doctors.

Secondly, the opportunities for private practice for hospital specialists in the NHS, and the independent contractor status of general practitioners, mean that the incentives facing doctors are different from those in Kaiser. In Kaiser there is a strong sense that doctors and the health plan are working to a common purpose and that doctors have a commitment to the success of the organisation.

Thirdly, the NHS is a universal service, but Kaiser is not. By focusing on the population aged 65 and over, who in the United States are all covered by Medicare, and by standardising the data on utilisation by age bands, we have sought to control for differences in population characteristics. Despite this, the comparison may still not be on a like for like basis because of evidence that older people enrolled in managed care plans use fewer resources than those served by fee for service schemes. ${ }^{6}$

\section{Conclusion}

The data we have analysed confirm that there is scope for acute hospital beds to be used differently in the NHS. ${ }^{7}$ Commenting on the analysis by Feachem and colleagues, Berwick argued that hospitals should regard an unneeded day of stay in hospital as a defect, ${ }^{8}$ and our results indicate the scope for addressing this defect. Specifically, the NHS can learn from Kaiser's approach by developing closer integration between primary and secondary care, making use of intermediate care, focusing on chronic diseases and their effective management, and giving priority to self care and the use of patients and families as co-providers. The NHS can also learn from Kaiser's experience of engaging doctors in developing and supporting an integrated model of care.

We are grateful for the help provided by Gerry Anderson, Bob Crane, Murray Ross, Julien Forder, Ian Stone, Kathy Lee, Clive Smee, Simon Stevens, Andrew Jackson, and John Reed in supplying data and commenting on earlier drafts of this paper.

Contributors: CH developed the idea for this study. NY, SS, and RS gathered and analysed the quantitative data, and $\mathrm{CH}$ undertook the qualitative study. CH led on the writing of the paper with contributions from NY, $\mathrm{SS}$ and RS. CH is guarantor.

Funding: No additional funding.

Competing interests: None.

Ethical approval: Not needed.

1 Feachem RGA, Sekhri N, White K. Getting more for their dollar: a comparison of the NHS and California's Kaiser Permanente. BMJ 2002;324:135-43.

2 Hospital episode statistics 2000-2001. London: Department of Health, 2001.

3 Audit Commission. Lying in wait: the use of medical beds in acute hospitals. London: HMSO, 1992.

4 Crosson FJ. Kaiser Permanente: a propensity for partnership. BMJ 2003;326:654.

5 Enthoven AC. Competition made them do it [commentary]. BMJ 2002;324:143.

6 Hellinger FJ, Wong HS. Selection bias in HMOs: a review of the evidence. Med Care Res Rev 2000;57:405-39.

7 Goddard M, McDonagh M, Smith D. Avoidable use of beds and cost-effectiveness of care in alternative locations. In: Department of Health. Shaping the future NHS: long term planning for hospitals and related services. London: Department of Health, 2000:96-101. planning for hospitals and related services. London: Department of Health, 2000:96-101.

8 Berwick D. Same price, better care [commentary]. BMJ 2002;324:142-3.

(Accepted 26 September 2003)

bmj.com 2003;327:1257

Strategy Unit, Department of Health, London SW1A 2NS Chris Ham director

Economics and Operational Research Division, Department of Health, Leeds LS2 7UE

Nick York senior economic adviser

Rob Shaw economic adviser

NHS Information Authority, Winchester SO22 5DH

Steve Sutch senior consultant and analytical and statistical services manager

Correspondence to C Ham

Chris.Ham@doh.gsi.gov.uk 